42

doi:10.1017/cjn.2018.283

\section{Use of Alisertib in an adult patient with recurrent atypical teratoid/thaboid tumor: A case report and literature review of AT/RT in adults}

Navya Kalidindi, Warren P Mason. navya.kalidindi@uhn.ca

Atypical Teratoid Rhabdoid Tumors (AT/RTs) often affect children under the age of 3 and are the most common malignant CNS tumors in children younger than 6 months. It is very rare to see these tumors in patients older than 6 years of age. We discuss the case of a 14 year old male with AT/RT of the right insula. He had a prior diagnosis of Dysembryoplastic Neuroectodermal Tumor (DNET) at the age of 10, after two years of intermittent headaches, nausea, and seizures, which was treated with conformal radiation and chemotherapy for a year. Following the diagnosis of $\mathrm{AT} / \mathrm{RT}$, he underwent radiotherapy, multiple lines of chemotherapy, and two additional debulking surgeries of the left temporal lobe due to continuing progression. He was then treated with Alisertib (an Aurora-A kinase inhibitor) with good response on sequential MRIs after the first three cycles. He progressed after nine cycles of Alisertib and required further debulking surgery. Six years after his AT/RT diagnosis (and 10 years after his DNET diagnosis), the patient expired at the age of 20 due to ongoing progression. To our knowledge, this is only the second reported case of Alisertib use in a non-pediatric AT/RT case. We also performed a literature review of all reported cases of AT/RT in adults between the years $2000-2017$ and discuss treatment options, patient demographics, and survival.

\section{4}

doi:10.1017/cjn.2018.284

\section{Success of stem cell collection and neutrophil recovery in pediatric patients with brain tumours}

George Michaiel, Tony Truong, Nicole Prokopishyn, Henry Luu, Lucie Lafay-Cousin, Doug Strother, Greg Guilcher and Victor Lewis. george.michaiel@ahs.ca

BACKGROUND: The use of high-dose chemotherapy (HDC) and autologous hematopoietic stem cell transplant (HSCT) has been used in certain pediatric patients with brain tumours to delay/spare radiotherapy. We aimed to study factors predicting a successful stem cell collection (SCC) and correlate stem cell dose infused with HSCT outcomes. METHODS: A retrospective chart review was undertaken for pediatric patients with brain tumours treated at our centre with HDC/HSCT between 2004-2016. RESULTS: Fifty-five patients were identified ( 32 male) with median age of 6.3 years at time of SCC (range 0.4-18.7). Patients' diagnoses were medulloblastoma (62\%), ATRT (20\%), and PNET (18\%). Most patients $(82 \%)$ underwent a single/1-day SCC, while the remaining required 2 SCC procedures. Peripheral blood stem cells were the source in most collections (95\%). Successful SCC (CD34 collected greater-than-or-equal-to $2 \times 10^{\wedge} 6 / \mathrm{kg} /$ transplant) and ideal SCC (greater-than-or-equal-to $5 \times 10^{\wedge} 6 / \mathrm{kg} /$ transplant) was achieved in $85 \%$ and $45 \%$ of patients, respectively. Use of mobilizing chemotherapy with G-CSF was the only factor associated with achieving an ideal collection, while gender, age, stem cell source, and pre-apheresis peripheral blood CD34 count were not significant. Higher CD34/kg infused was associated with faster neutrophil engraftment in the first 3 courses of HDC/HSCT and platelet engraftment in the first course. CONCLUSIONS: The majority of SCC for autologous HSCT can be successfully completed with a single apheresis session. Mobilization with both chemotherapy and G-CSF yields higher CD34 compared to G-CSF alone. Higher dose of CD34/kg infused was associated with faster neutrophil and to a more limited scale platelet recovery postHSCT.

\section{5}

doi:10.1017/cjn.2018.285

Cognitive rehabilitation in neuro-oncology: Program development and evaluation

NM Richard, WP Mason, D Shultz, N Laperriere, BA Millar, A Berlin, F Moraes, T Conrad, M Patel, C Maurice, M Bernstein, $P$ Kongkham, G Zadeh, LJ Bernstein, K Edelstein. nadine.richard@uhn.ca

Background: Brain tumors present unique challenges to patient and family quality of life (QOL). Cognitive dysfunction is common and functionally limiting, with no established treatments. These studies evaluate feasibility and preliminary efficacy of behavioral interventions developed for neuro-oncology patients. Study 1: A randomized controlled trial $(\mathrm{N}=25$ primary brain tumor patients) compared an adapted version of Goal Management Training (GMT, a neuroscience-based integration of mindfulness and strategy training) and a newly-designed supportive psychoeducational intervention (Brain Health Program, BHP) to standard of care. Each intervention comprised 8 individual sessions and at-home practice between sessions. GMT patients" executive functions improved immediately $(\mathrm{p}=.077, \mathrm{~d}=1.13)$, with maintenance at 4-month follow-up $(p=.046, d=1.09)$. Both intervention groups reported improvements in everyday cognitive functioning immediately $\left(\mathrm{p}=.049\right.$; $\left.\mathrm{d}^{\text {ee }} \mathrm{s} \mathrm{GMT}=0.43, \mathrm{BHP}=0.79\right)$ and at follow-up $(\mathrm{p}=.001$; des $\mathrm{GMT}=0.22, \mathrm{BHP}=1.01)$. BHP patients also reported improved $\operatorname{mood}\left(\mathrm{p}^{\text {ec }} \mathrm{s}=.026 \& .012, \mathrm{~d}^{\mathrm{ee}} \mathrm{s}=0.61 \& 0.62\right)$. Study 2: Following a needs assessment about cognitive concerns and QOL in brain metastases patients $(\mathrm{N}=109)$ and caregivers $(\mathrm{N}=31)$, we developed a novel, brief (3 sessions + homework) Cognitive Support Program to provide education and strategytraining in key areas of concern: executive functions, memory, and communication. Options include caregiver co-training, and inperson or web-based delivery. Preliminary data from a pilot trial in progress demonstrate objective and subjective improvements. Conclusions: Cognitive rehabilitation may be a feasible and effective option for primary or metastatic brain tumor patients, addressing a need that is largely unmet in standard cancer care. Further development and larger trials appear warranted, with capacity for remote delivery recommended.

\section{7}

doi:10.1017/cjn.2018.286

Preclinical studies of dianhydrogalactitol (VAL-083) in DIPG, as single agent or as a combination with AZD1775 or radiation

Xiaodong Yang, Anne Steino, Jeffrey Bacha, Dennis Brown, Sabine Mueller. asteino@delmarpharma.com 
Despite decades of trials, the prognosis for diffuse intrinsic pontine gliomas (DIPG) remains dismal. DIPG is inoperable and standard treatment is radiation alone, as the addition of chemotherapeutic agents, such as temozolomide, have not improved survival. In addition to inherent chemoresistance, treatment of DIPG is impeded by an intact blood-brain barrier (BBB). VAL-083 is a structurally unique bi-functional DNA-targeting agent that readily crosses the BBB. VAL-083 forms interstrand DNA crosslinks at N7-guanine, resulting in DNA double-strand breaks (DSB), S/G2phase cell-cycle arrest, and ultimately cancer cell death. We have previously demonstrated that VAL-083 is able to overcome temozolomide-resistance in vitro and in vivo, and that its cytotoxicity is independent of the DNA-repair enzyme O6methylguanine DNA-methyltransferase (MGMT). MGMT is almost universally expressed in DIPG and its expression is strongly correlated with temozolomide-resistance. VAL- $083^{\text {ee }} \mathrm{s}$ distinct mechanism-of-action suggests the potential for combination with inhibitors of DNA DSB repair or S/G2 cell-cycle progression (e.g. Wee1 inhibitor AZD1775). Here, we investigated the effects of VAL-083 in combination with radiation, AZD1775 or irinotecan (topoisomerase inhibitor) in three DIPG cell-lines: SF10693 (H3.1), SF8628 (H3.3) and NEM157 (H3.3). VAL-083 showed activity at low $\mathrm{uM}$-concentration in all three cell-lines. In addition, VAL-083 showed synergy with AZD1775 in all three cell-lines. Combined with its ability to cross the BBB, accumulate in brain tumor tissue and overcome MGMT-related chemoresistance, these results suggest VAL-083 as a potentially attractive treatment option for DIPG as single agent or in combination with AZD1775. Combination studies with radiation are ongoing and will be presented at the meeting.

\section{8}

doi:10.1017/cjn.2018.287

\section{Phase 2 studies of dianhydrogalactitol (VAL-083) in patients with glioblastoma, MGMT-unmethylated}

JA Bacha, A Steino, R Schwartz, J Langlands, S Kanekal, LM. Lopez, BJ O'Brien, ZP Chen, M Penas-Prado, DM Brown. asteino@delmarpharma.com

Current standard-of-care for glioblastoma (GBM) includes surgery, radiation and temozolomide. Most tumors recur within a year from diagnosis and median survival for recurrent GBM (rGBM) is 3-9 months. Unmethylated promoter status for O6-methylguanineDNA-methyltransferase (MGMT) is a validated biomarker for temozolomide-resistance, exhibited by most GBM patients. VAL083 is a DNA-targeting agent with a mechanism-of-action that is independent of MGMT. VAL-083 overcomes temozolomideresistance in GBM cell-lines, cancer stem cells, and in vivo models. VAL-083 readily crosses the blood-brain barrier and accumulates in brain-tumor tissue. We recently completed a VAL083 dose-escalation trial in temozolomide- and bevacizumabrefractory $\mathrm{rGBM}$ and determined that $40 \mathrm{mg} / \mathrm{m} 2 /$ day given intravenously on days $1,2,3$ of a 21-day cycle is generally welltolerated. This dosing regimen was selected for subsequent GBM trials, including an ongoing single-arm, biomarker-driven Phase 2 trial $(\mathrm{N}=48)$ in temolozomide-refractory, bevacizumab-naïve rGBM , MGMT-unmethylated (Clinicaltrials.gov:NCT02717962). The primary objective of this study is to determine if VAL-083 improves OS compared to a historical control of 7.15 months for MGMT-unmethylated rGBM patients treated with lomustine (EORTC26101). In addition, another single-arm, biomarker- driven, Phase 2 study $(\mathrm{N}=25)$ of VAL-083 in combination with radiotherapy in newly diagnosed GBM, MGMT-unmethylated is ongoing (Clinicaltrials.gov:NCT03050736). This trial aims to determine a dose for further study of VAL-083 in combination with radiotherapy and explore if VAL-083 improves PFS and OS compared to historical results in newly diagnosed GBM. Enrollment and safety data updates will be provided at the meeting. The results of these studies, if successful, may support VAL-083 as part of a new chemotherapeutic treatment paradigm for GBM.

\section{0}

doi:10.1017/cjn.2018.288

\section{Are gangliogliomas in children and adults disorders of nervous system development?}

Qiang Jiang, Jamie Zagozewski, Paolo Nozza, Beverly Wilson, Frank Van Landeghem, David Eisenstat. qiiang@ualberta.ca

INTRODUCTION: Gangliogliomas (GGs) are neuroepithelial tumours of the central nervous system (CNS) composed of mature ganglion cells or a mixed population of ganglion and glial cells. Microarray data of low grade gliomas (LGG) including GGs revealed overexpression of the Dlx2 gene, a homeobox gene essential for interneuron migration and differentiation. We hypothesized that GGs are arrested in development, and began to explore the role of the Dlx2 gene. BRAF rearrangements and BRAF V600E point mutations have been reported in pediatric LGG. METHODS: DLX2 expression was examined in GGs using immunofluorescence (IF) and immunohistochemistry (IHC) labelling of formalin fixed paraffin embedded (FFPE) tissue sections, along with staining of glial and neuronal markers. BRAF mutations were detected using a commercial antibody and/or sequence verification of the DNA extracted from the FFPE blocks. RESULTS: In the Discovery cohort 10/30 were DLX2+ (33.3\%) and in the Validation cohort $15 / 40$ were DLX2+ (37.5\%). Of these 15 cases, 15 were GFAP+ (100\%), 15 were synaptophysin and/or $\mathrm{NeuN}+(100 \%)$, and 13 were OLIG2+ $(86.7 \%) ; 6$ had a BRAF V600E mutation $(40.0 \%)$. For the Validation cohort of 40 GGs, 28 were OLIG2+ (70.0\%); 13/28 co-expressed DLX2 (46.4\%). 18/40 cases had a BRAF V600 mutation(17 V600E, 1 V600G; 45.0\%) and $6 / 18$ were DLX2+ $(33.3 \%)$. CONCLUSIONS: DLX2 is expressed in GGs in both neuronal and glial marker expressing tumour cells. Our results support that GGs arise from CNS progenitors arrested at the neuronal-glial cell fate "decision" point.

\section{1}

doi:10.1017/cjn.2018.289

\section{Functional characterization of ribosomal RNA methyltransferase NSUN5 in glioblastoma}

\section{Jiesi Zhou*, Krista Vincent, Scott Findlay, Daniel Choi, Roseline Godbout, Lynne-Marie Postovit, YangXin Fu. jiesi@ualberta.ca * CRINA Travel Award Recipient}

Glioblastoma is the most common and malignant brain tumor with a median overall survival of 20.5 months. There is an urgent need to develop novel therapeutic strategies. Using a glioblastoma TCGA dataset, we have determined that high NSUN5 mRNA expression is strongly associated with poor survival in glioblastoma patients. NSUN5 is a ribosomal RNA (rRNA) 